\title{
BMJ Open Quality Using lean management approach in improving clinical team leader handover process: nursing services
}

\author{
Lina Mohammed Obaid, Ahmad Al Baker, Jessalie Ann Husain, Gretchen Cabania, \\ Sherryl Roque
}

To cite: Obaid LM, Al Baker A, Husain JA, et al. Using lean management approach in improving clinical team leader handover process: nursing services. BMJ Open Quality 2021;10:e001375. doi:10.1136/ bmjoq-2021-001375

- Additional supplemental material is published online only. To view, please visit the journal online (http://dx.doi.org/10. 1136/bmjoq-2021-001375).

Received 28 January 2021 Accepted 18 July 2021

\section{ABSTRACT}

This report describes the result of the study using lean management approach in improving clinical team leader handover process in nursing services at Sultan Bin Abdulaziz Humanitarian City, the largest rehabilitation facility in the Middle East with 511-bed capacity and more than 20 nursing inpatient units. Clinical handover refers to the transfer of professional responsibility and accountability for some or all aspects of care for a patient, or group of patients, to another pehealthcare system is a crson or professional group on a temporary or permanent basis. It is in fact a valuable and essential part of the care processes in the hospitals. However, clinical team leaders face a challenging role during handover due to prolonged process, causing extra nursing working hour beyond their 12-hour scheduled shift, resulting in additional burden and exhaustion. The aim of this project was to test the effectivity of the lean management approach in improving the duration of clinical handover by reducing the handover time frame to $50 \%$ through eliminating unnecessary steps towards a more efficient, sustainable and effective communication between clinical nursing team leaders. The project results demonstrated the effectiveness and efficiency of the team leader clinical handover process by decreasing the duration by $50 \%$. One hundred per cent of nursing units that were involved in the project were able to start and end their team leader handover process by the average of the selection target of 20-30 min of handover duration.

\section{INTRODUCTION}

Nursing services in Sultan Bin Abdulaziz Humanitarian City's vision is to be recognised as a leading specialised nursing service in rehabilitation and acute care, regionally and globally, which led us to use all resources and evidence to improve nursing practice and processes in the facility.

Through our partnership with the National Data Base of Nursing Quality Indicators (NDNQI), the department was able to identify one area for improvement which is related to clinical handover process between nursing team leaders.

In the last RN Satisfaction Survey-NDNQI in $2019,83.2 \%$ of the staff reported that they are working an extra 1 hour beyond their 12-hour shift to complete the patient handover. This caused additional burden and exhaustion on their role as the nurse team leader of the shift.

This finding prompted the team to formulate a core group to investigate and carry out a systematic and formal inquiry to discover and examine the facts of the incident.

Using all resources and tools are improving nursing practices and processes in any healthcare organisation ${ }^{1}$; moreover, improving communication process between healthcare providers was declared as the international goal of patient safety by Joint Commission International. $^{2}$

The effective communication between healthcare providers as well as the clinical handover team will improve the safety and quality of the patient experience. ${ }^{3}$

Clinicians should follow wellcommunicated protocols that guide care and communication $^{4}$ as the healthcare system is a complex system; thus, the wellcommunicated protocols and guidelines will decrease the likelihood of mistake occurring and medical errors from reaching the patients.

\section{METHODS}

After formulating the team, performance improvement practicum was conducted for 2 days to educate each member about the principles and steps of lean management. The roles and responsibilities of each member were also identified parallel with the project charter.

Project charter is a formal and short document that describes the project in its entirety ${ }^{5}$-including the goals and objectives, project scope, inclusion and exclusion criteria, milestones, stakeholders as well as the quality methodology.

The first step of the project commenced by engaging front-line staff through conducting staff interview sessions titled with 'Hearing 
the Staff Voice' to get feedback on the prolonged clinical handover process. Fifty per cent of nursing units were visited, and based on team's observations and staff responses, the extra 1 hour beyond their 12-hour shift is due to the long handover process between clinical team leaders.

Following staff interview, the team proceeded with gemba walk, ${ }^{6}$ where each member attended and observed the handover process in real time in all nursing inpatient units, noting the following:

1. Duration of handover.

2. Types of waste or unnecessary information or data.

3. Types of defect or gaps in the process.

4. Common practices and handover tool used in all areas. About $50 \%$ of all areas were included in the gemba walk, and a total of 12 handover processes were attended by the team. On completion, the team found out that all units have an average of 40-60 min to finish the handover process (online supplemental file 1), which is beyond the selected average target of $20-30 \mathrm{~min}$ of the regular clinical handover.

The team adapted the value stream mapping tool from the lean management approach to analyse what is currently happening (the current state) and goes on to design a future state for the process focusing on how value is created and delivered to the patient or staff. Value stream mapping as seen by many lean practitioners is a fundamental tool to identify waste, reduce process cycle times and implement process improvement.

\section{Value adding, non-value-adding and necessary but non- value-adding steps}

During this phase, the team designed the value stream mapping in each unit by (1) involving all patients, (2) considering not only the flow of the patient handover but also the flow of other information needed, (3) walk through the handover process from beginning until the end and (4) draw the process with the starting point until the end point. After the map was completed, the team determined what adding value to process and what did not. However, there are some steps that have no additional value yet are still necessary. Drawing the map is reflecting the longest step of the process as well as those patient handovers with more minutes than others (figure 1).

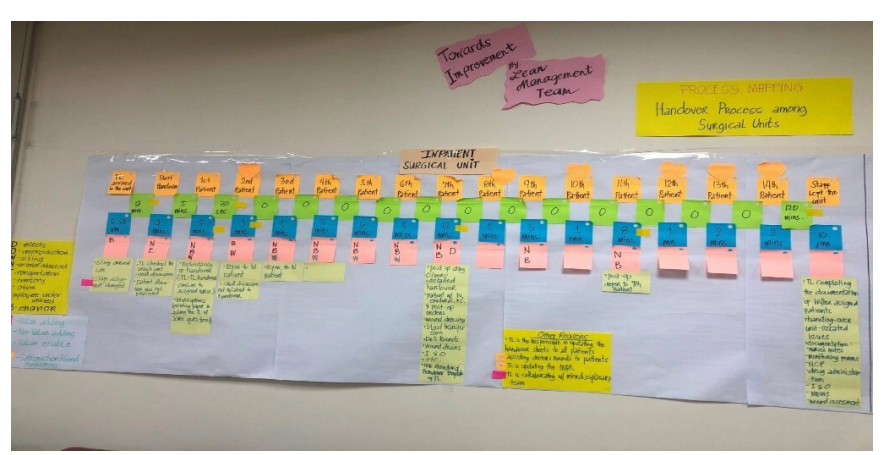

Figure 1 Value stream mapping.

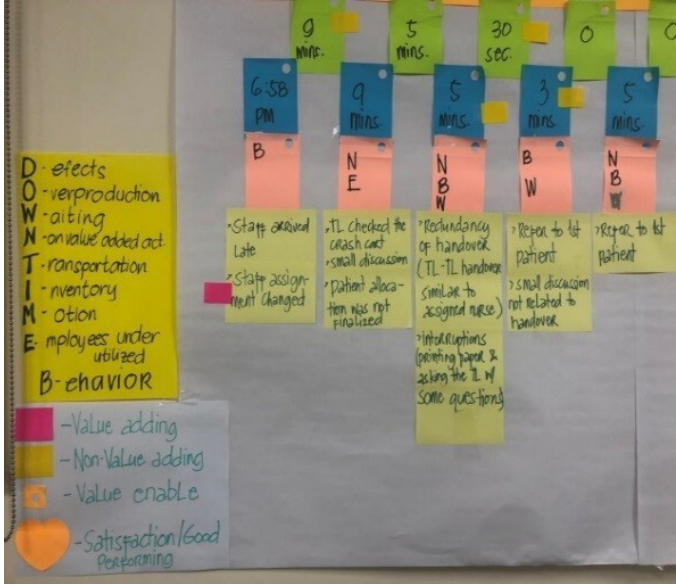

Figure 2 Acronym of DOWNTIME-eight kinds of waste.

Symbols were used in order to denote the value of a step, such as sticky dots/notes (pink for value adding, yellow for non-value adding and orange dot for non-value adding but essential step 'value enable', and orange heart for satisfactory/good performing step) (figure 2). Moreover, the team identified the type of waste by referring to the eight kinds of waste, in an acronym DOWNTIME.

1. Defect.

2. Overproduction.

3. Waiting.

4. Non-value-adding act.

5. Transportation.

6. Inventory.

7. Motion.

8. Employee underuse.

In addition, $B$ as a waste related to behaviour.

After finalising the value stream map, five kinds of waste were identified by the project members, affecting the handover process time frame among all nursing inpatient units. These are non-value-adding act, behaviour, waiting, defect and employee underuse.

\section{TARGET SELECTION}

Before the implementation of the new interventions, the team reviewed the handover time and duration in order to set a target level before measuring the process. The target level will support the project in standardising the handover duration in all units to ensure that the staff are not exceeding their scheduled 12 hour per shift due to long handover process. After the review, the identified average of handover duration was between 20 and $30 \mathrm{~min}$.

Our baseline measurement helped us identify major contributing factors for the prolonged handover such as

- No standardised practice among nursing units relating to team leader handover.

- No standardised assignment regarding the team leader role and function at the beginning of each shift.

- Redundancy of team leader handover information, which is similar to the assigned nurse handover. 
- Detailed handover with 'unnecessary information' being mentioned which is not related to team leader role and function.

- Many external interruptions were happening during the process itself, such phone calls and physician morning visit.

- Lengthy time of narcotic checking due to high volume of narcotic and controlled medication stocks in some units, delaying the handover.

The team generated a list of possible solutions through structured brainstorming sessions.

More than 15 solutions were listed and sorted using prioritisation matrix tool, taking into consideration the impact and control from high, medium and low levels.

Planned interventions

- To create a standard template for team leader handover process.

- Engage unit team leader in creating the new form of team leader handover sheet.

- Use ISBAR standard requirement while building the team leader form.

- To standardise the role and function of the team leaders beginning of the shift.

- Eliminate the interruption during the handover by designing an 'ongoing team leader handover signage' to guide the other interdisciplinary team not to interrupt the ongoing handover.

- Revise the assignment sheet of the staff and allocate staff to answer patient calls and phone calls.

- Review the narcotic and controlled medication list in each unit in order to remove the non-moving and prioritise the slow-moving and fast-moving medications. Delegate narcotic checking to an eligible staff other than the team leader.

The success of this project led to the development of a standardised team leader sheet (online supplemental file 2) by using the ISBAR communication $\operatorname{tool}^{7}$ as a guidance. Several sessions were conducted before building the sheet in order to get and collect feedback from team leaders among all nursing units. Further to this, staff engagement will enhance the efficiency and effectiveness of the form as it will be derived from their specific needs and experience. ${ }^{78}$

The new sheet was designed to cover two essential parts for the vital information related to the unit and patient's condition.

\section{Unit-supported information}

This includes total census, total sitters, total in-house, total admission, total discharge, total trans-in, total trans-out, total hospital acquired pressure injury, total community acquired pressure injury, total patient with orthosis, and name of team leaders for in/out shift.

\section{Clinical-supported information}

Patient information

- Name, medical record number, date of admission, date of discharge, diagnosis, surgery date, primary physician.

Situation and background

Current condition.

- Blood sugar.

- Vital signs.

Special notes.

- Free textbox.

\begin{tabular}{|lrrrrr}
\multicolumn{5}{c}{ The Time-frame of Handover Process among Nursing } \\
& \multicolumn{1}{c}{ Unit \#1 } & Unit \#2 & Unit \#3 & Unit \#4 \\
\multirow{4}{*}{ Pre } & 1st Visit & 60 & 63 & 55 & 40 \\
& 2nd Visit & 50 & 60 & 70 & 35 \\
& 3rd Visit & 45 & 65 & 50 & 37 \\
& 4th Visit & 35 & 38 & 40 & 30 \\
& 5th Visit & 20 & 20 & 17 & 25 \\
Post & 6th Visit & 25.5 & 17 & 25.5 & 23 \\
& 7th Visit & 27.5 & 19 & 20 & 17 \\
& 8th Visit & 30 & 20 & 15 & 15
\end{tabular}

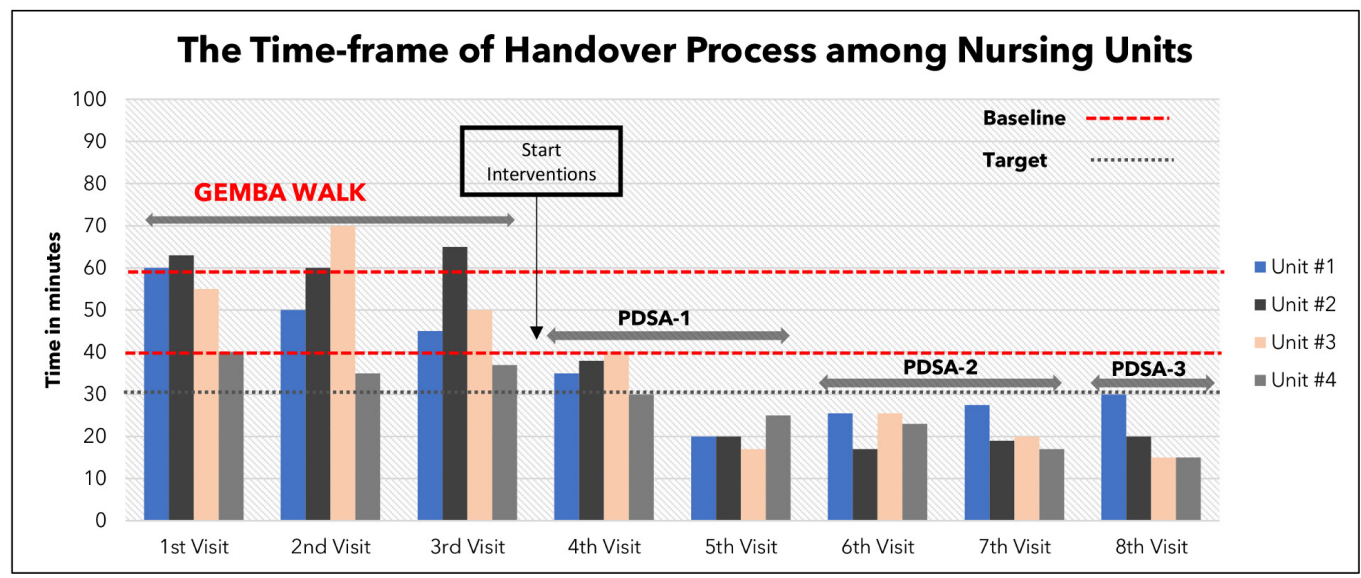

Figure 3 Turnaround time of handover process among nursing units. 
Table 1 (P. Value - Pre Interventions \& Post Interventions)

\begin{tabular}{lcc}
\hline & Pre-interventions & Post-interventions \\
\hline Mean & 52.5 & 25.5 \\
Variance & 122.7037037 & 5.166666667 \\
Observations & 4 & 4 \\
Pearson correlation & 0.121354568 & \\
Hypothesised mean difference & 0 & \\
df & 3 & \\
$t$ stat & 4.893761063 & \\
$P(T \leq t)$ one-tail & 0.008162026 & \\
$t$ critical one-tail & 2.353363435 & \\
\hline$P(T \leq t)$ two-tail & 0.016324052 & \\
\hline$t$ critical two-tail & 3.182446305 & \\
\hline
\end{tabular}

Assessment (finding)/current issues

- Presence of contraptions (intravenous, epidural, PCA, catheter, drains).

- Lab result.

- Diagnostic imaging result.

- Allergy.

- Wound dressing.

Recommendation

- Referral to

Consultation.

Nurse specialties (WCS - Wound Care specialist, CNS - Continence Nurse specialist, DNS - Diabetic Nurse Specialist, IPC - Infection control and prevention team Case manager

Another interdisciplinary team.

- Reminder for follow-up action.
Need to be followed up by house physician

Need to be followed up by psychiatrist.

Appointment.

In addition to standardising the team leader handover sheet, the team leader's role and responsibilities were outlined, and non-adding value tasks were eliminated to allow them to focus on the patient handover and to avoid unnecessary interruptions. Additionally, the most senior staff of the incoming shift or the designated nurse-in-charge of the shift was identified as the key leader in the handover process. Non-adding value tasks were delegated to other competent nurses. Tasks were appropriately distributed in terms of allocating it to those with the skills to undertake them most effectively in the patient's best interests and to ensure the best use of the available team member's time with consideration

\begin{tabular}{|c|c|c|c|c|c|}
\hline \multicolumn{2}{|l|}{ Unit \# } & \multicolumn{2}{|l|}{ Preintervention } & \multicolumn{2}{|l|}{ Postintervention } \\
\hline Mean & 2.5 & Mean & 52.5 & Mean & 25.5 \\
\hline SE & 0.645497224 & SE & 5.538585192 & SE & 1.136515141 \\
\hline Median & 2.5 & Median & 55 & Median & 26 \\
\hline Mode & \#N/A & Mode & $\# N / A$ & Mode & \#N/A \\
\hline SD & 1.290994449 & SD & 11.07717038 & SD & 2.273030283 \\
\hline Sample variance & 1.666666667 & Sample variance & 122.7037037 & Sample variance & 5.166666667 \\
\hline Kurtosis & -1.2 & Kurtosis & 0.836214643 & Kurtosis & -0.747658689 \\
\hline Skewness & 0 & Skewness & -1.098677553 & Skewness & -0.894074346 \\
\hline Range & 3 & Range & 25.33333333 & Range & 5 \\
\hline Minimum & 1 & Minimum & 37.33333333 & Minimum & 22.5 \\
\hline Maximum & 4 & Maximum & 62.66666667 & Maximum & 27.5 \\
\hline Sum & 10 & Sum & 210 & Sum & 102 \\
\hline Count & 4 & Count & 4 & Count & 4 \\
\hline $\begin{array}{l}\text { Confidence level } \\
(95 \%)\end{array}$ & 2.054260257 & $\begin{array}{l}\text { Confidence level } \\
(95.0 \%)\end{array}$ & 17.62624998 & $\begin{array}{l}\text { Confidence level } \\
(95.0 \%)\end{array}$ & 3.616898413 \\
\hline
\end{tabular}

\# means number of units.

N/A, Not applicable. 


\begin{tabular}{|c|c|c|c|}
\hline SN & Area & Calculation & Remarks \\
\hline 1 & Target cost saving & $\begin{array}{l}\text { Working hrs for surgical unit's team } \\
\text { leader }\end{array}$ & \\
\hline 2 & Number of surgical units team leader & $\begin{array}{l}5 \text { units } \times 5 \text { team leaders }=25 \text { team } \\
\text { leaders }\end{array}$ & $\begin{array}{l}\text { Each team leader works for } 16 \\
\text { shifts per month }=192 \text { hours } / \text { month. }\end{array}$ \\
\hline 4 & $\begin{array}{l}\text { Average saved time each shift } \\
\text { endorsement }\end{array}$ & $40 \mathrm{~min}$ & \\
\hline 5 & Total saved time & $\begin{array}{l}40 \mathrm{~min} \times 16 \text { shifts } \times 25 \text { team } \\
\text { leaders }=16000 \mathrm{~min}=267 \text { working hours }\end{array}$ & \\
\hline 6 & Total worth cost saving & $\begin{array}{l}267 \text { hours } \times 31.25 \mathrm{SR}=8343 \mathrm{SR} / \\
\text { month } \approx \$ 2224.5 / \text { month }\end{array}$ & \\
\hline
\end{tabular}

given to overall workload and other demands on the team.

\section{STRATEGY}

Implementation: PDSA (Plan, Do, Study, \& Act) cycles

In improving the process of clinical handover among team leaders, the core group used the PDSA tool-a fourstage problem-solving model. Initially, the team started with small scales in each cycle by selecting four adult inpatient units with different patient types for piloting the new process. This will help the team and the selected units to tackle any issues or problems that may arise while implementing the proposed actions. This will also give an opportunity to improve the intervention before moving to the next cycle as well as to ensure the effectivity of interventions prior to disseminating to all nursing units.

\section{PDSA cycle 1}

The first cycle started by introducing a new team leader handover sheet to the selected units for piloting. To ensure staff awareness on the new form, two educational sessions were held to orient them on the new process with their outlined specific tasks.

During this phase, project team members joined the unit clinical handover to observe and examine the use of the new team leader handover sheet, noting the implementation of the changes and the presence of any nonvalue-added tasks, unnecessary waiting time, defect in the new process and employee underuse.

After attending two sessions of the clinical handover in each piloted unit, the team noticed a remarkable $30 \%-40 \%$ decrease in the duration of the handover process. Moreover, there were no significant issues encountered during this phase, and unit staff were satisfied on the outcome of the new team leader handover sheet.

\section{PDSA cycle 2}

Apart from the new team leader handover sheet developed in PDSA cycle 1, the team also introduced the new signage of 'ongoing team leader handover'. This signage is written in Arabic and English languages to prevent interruptions and unnecessary distractions during the handover. In addition to this, the unit initiated the revised assignment sheet, which aimed to assign one staff to answer patient call bells and phone calls during the handover; those actions were implemented parallel to the new handover sheet. While implementing the new interventions on this cycle, the project team joined the handover process during this PDSA cycle to monitor and evaluate the progress.

Furthermore, the new signage provided a significant improvement by avoiding and eliminating any interruptions which resulted to an average handover process duration of $30 \mathrm{~min}$ among piloted units.

\section{PDSA cycle 3}

The team expanded the piloted unit to add two more units in order to implement those interventions under cycles 1 and 2. As narcotic and controlled medication checking is one the identified factors prolonging the clinical handover process, the team worked closely with pharmacy section in order to remove the non-moving medications at the narcotic cabinets and to prioritise the slowmoving and fast-moving medications-this intervention helped the team leaders to save and maximise their time while checking the controlled and narcotic medication cabinet. Furthermore, this task was delegated to another registered nurse eligible to handle the team leader role but is not assigned on that shift as team leader. This cycle was able to sustain the improvement of the first and second cycles by keeping the duration average of handover between 20 and $30 \mathrm{~min}$ among all piloted units.

\section{RESULT: PROJECT OUTCOMES}

The team examined the result of PDSA cycles 1-3 for quantifiable outcomes among the four piloted units. Figure 3 shows the significant improvement in the average time frame of the team leader handover process using histogram chart.

In addition, in the calculation of handover duration for the piloted unit, statistical data were used to support the outcome of the project. The following tables reflect that the duration of handover is statistically significant 
between the preinterventions and postinterventions through calculating the $\mathrm{p}$ value $(\mathrm{p}=0.008)$ (table 1$)$ with the CI of $95 \%$ (table 2).

\section{Impact on efficiency: staff working hours}

Generally, saving healthcare providers' time equals cost saving. The following calculation (table 3) shows the efficiency of staff working hours, which directly impacts the total saved time and the total cost saved. In conclusion, saving an extra $40 \mathrm{~min}$ in the team leader handover process will save 8343 saudi riyal per month, which equal to $\$ 2224.5$ that the organisation shall pay to the staff.

\section{LIMITATION AND LESSONS}

One of the limitations of this project is that the team was not able to use the system in order to create electronic handover. The reason is that the current health information system is not supporting the group handover of patients and it was not built to have a team leader handover electronic sheet. Furthermore, due to the short period of the project, the team members were not able to gather more data points, which limited them from considering the control chart as a sustainability measurement.

The team list major lessons learnt that were added a value based on the project outcomes such as

- Having a standard practice will avoid any variation between units and making the flow easier.

- Engaging front-line staff as the end users of the team leader handover process cannot be understated, as this helps to ensure the sustainability of the new process.

- Improving the leadership skills such as time management and assertiveness will help team leaders to lead the unit and to end the shift effectively.

- Teamwork avoids delay in the process and in patient care, and the team will achieve the desired objective.

\section{CONCLUSION}

Lean management approach is effective in improving the duration of clinical handover by eliminating unnecessary steps towards a more efficient, sustainable and effective communication between clinical team leaders.
Acknowledgements The authors thank the staff at the Quality Management Department, Sultan Bin Abdulaziz Humanitarian City.

Contributors LMO, JAH, SR and GC contributed to the design and implementation of the project, the analysis of the results and the writing of the manuscript.

Funding The authors have not declared a specific grant for this research from any funding agency in the public, commercial or not-for-profit sectors.

Competing interests None declared.

Patient consent for publication Not applicable.

Ethics approval This report was deemed a clinical improvement project and therefore did not require local ethical approval.

Provenance and peer review Not commissioned; externally peer reviewed.

Data availability statement All data relevant to the study are included in the article or uploaded as supplementary information.

Supplemental material This content has been supplied by the author(s). It has not been vetted by BMJ Publishing Group Limited (BMJ) and may not have been peer-reviewed. Any opinions or recommendations discussed are solely those of the author(s) and are not endorsed by BMJ. BMJ disclaims all liability and responsibility arising from any reliance placed on the content. Where the content includes any translated material, BMJ does not warrant the accuracy and reliability of the translations (including but not limited to local regulations, clinical guidelines, terminology, drug names and drug dosages), and is not responsible for any error and/or omissions arising from translation and adaptation or otherwise.

Open access This is an open access article distributed in accordance with the Creative Commons Attribution Non Commercial (CC BY-NC 4.0) license, which permits others to distribute, remix, adapt, build upon this work non-commercially, and license their derivative works on different terms, provided the original work is properly cited, appropriate credit is given, any changes made indicated, and the use is non-commercial. See: http://creativecommons.org/licenses/by-nc/4.0/.

\section{REFERENCES}

1 Australian Medical Association. Safe handover: safe patients. AMAClinical handover guide. Sydney: AMA, 2006. http://ama.com.au/ node

2 Joint Commission international, 2021. Available: https://www.jointcom missioninternational.org/standards/international-patient-safety-goals/

3 Institute for Healthcare Improvement. SBAR communication technique. IHI, 2015. Available: http://www.ihi.org/Topics/SBARComm unicationTechnique/Pages/default.aspx

4 Prevention ME, Thomas L. Rodziewicz1; Benjamin Houseman2; John E. Hipskind3, 2020. Available: https://www.ncbi.nlm.nih.gov/books/ NBK499956/

5 Project charter. International Journal of Scientific \& Engineering Research 2015;6:856.

6 Womack JP, Walks G. Lean enterprise Institute, 2011.

7 Quality, service improvement and redesign tools: SBAR communication tool - situation, background, assessment, recommendation. Available: https://improvement.nhs.uk/resources/ sbar-communication-tool/

8 Plan-Do-Study-Act (PDSA) Worksheet. Available: http://www.ihi.org/ resources/Pages/Tools/PlanDoStudyActWorksheet.aspx 